\title{
Circulating HIV-1 Integrase Genotypes in Tanzania: Implication on the Introduction of Integrase Inhibitors-Based Antiretroviral Therapy Regimen
}

\author{
Salim Masoud, ${ }^{1}$ Doreen Kamori,, Godfrey Barabona, ${ }^{2}$ Macdonald Mahiti, \\ Bruno Sunguya, Eligius Lyamuya, and Takamasa Ueno ${ }^{1,2}$
}

\begin{abstract}
Tanzania has recently adapted World Health Organization antiretroviral guidelines that include integrase strand transfer inhibitors (INSTIs) in the first-line regimen. However, there is lack of evidence on integrase (IN) gene polymorphisms in viral strains circulating in Tanzania. In this study, we characterize IN gene polymorphisms in viral strains circulating in Dar es Salaam, Tanzania, before introduction of INSTIs. Plasma viral RNAs were prepared from 158 HIV-1-infected subjects, including 111 treated, but viremic (INSTI-naïve), subjects. A part of the pol gene encompassing the IN-coding region was amplified and directly sequenced by the Sanger sequencing method. Subtype analysis revealed that subtypes A1, C, and D and intersubtype recombinants were $42 \%, 38 \%, 11 \%$, and $9 \%$, respectively. Although multiple subtypes cocirculate, the IN gene exhibited a relatively conserved amino acid sequence pattern with an average Shannon entropy score of 0.16. No major INSTI resistance mutations were found; however, accessory resistance mutations at positions T97A, E157Q, $\mathrm{G} 163 \mathrm{E} / \mathrm{K}$, and $128 \mathrm{~A} / \mathrm{T}$ were detected in $5 \%$ of subjects.
\end{abstract}

Keywords: HIV-1, integrase, resistance, mutations, antiretroviral therapy

A NTIRETROVIRAL THERAPY (ART) has been effective in reducing the incidence of new infections and the burden of AIDS-related mortality and morbidity worldwide. However, HIV drug resistance presents a major challenge to the effectiveness of ART programs. In sub-Saharan Africa, high rates of pretreatment and acquired drug resistance to the nonnucleoside reverse transcriptase inhibitor (NNRTI)-based first-line regimen have been reported. ${ }^{1,2}$ Because of this situation, World Health Organization currently recommends the transition to the integrase strand transfer inhibitor (INSTI)-based first-line regimen, especially in countries where pretreatment resistance to NNRTI reaches $10 \%$.

In PEPFAR-funded ART programs in sub-Saharan African countries, the goal is to switch to a dolutegravir-based first-line regimen by 2021 with a fixed combination of dolutegravir, tenofovir, and lamivudine. This combination is not only expected to improve the level of viral suppression but it is also costeffective compared with the NNRTI-based first-line regimen because a less active pharmaceutical ingredient is required. ${ }^{3}$

Another merit of dolutegravir is that it has demonstrated a high genetic barrier to resistance in vivo. ${ }^{4}$ However, in sub-
Saharan African countries, factors such as poor adherence, inadequate virological monitoring, and lack of drug resistance testing increase the risk of acquiring drug resistance to dolutegravir. Indeed, after a few years of dolutegravir use in some African countries, dolutegravir resistance has already been reported in patients with quadruple-class resistance, such as in Botswana. ${ }^{5}$ This observation suggests that to maximize the potential benefits of the dolutegravir-based first-line regimen, vigilant drug resistance surveillance and treatment monitoring are necessary in sub-Saharan African countries, including Tanzania.

In Tanzania, since early 2019, newly diagnosed individuals and patients on the NNRTI first-line regimen were initiated on or switched to the dolutegravir + tenofovir + lamivudine fixed combination. This transition of regimen is done without drug resistance testing or prior confirmation of virological suppression, which opens a possibility of dolutegravir monotherapy when virological failure patients with resistance to the NRTI backbone (i.e., tenofovir + lamivudine) are involved. This approach may therefore pose a unique setting for development of dolutegravir resistance

\footnotetext{
${ }^{1}$ Department of Microbiology and Immunology, Muhimbili University of Health and Allied Sciences, Dar es Salaam, Tanzania.

${ }^{2}$ Division of Infection and Immunity, Joint Sciences Research Center for Human Retrovirus Infection, Kumamoto University, Kumamoto, Japan.
} 
different from high-income countries where reported resistance to dolutegravir has been low.

On the other hand, some viral factors, including naturally arising polymorphisms that affect sensitivity to INSTIs and HIV subtype-dependent differences in backbone sequences and codon usage, may confer increased risk of development of dolutegravir resistance. ${ }^{6}$ Therefore, this suggests that the presence of these viral factors and Tanzania's approach in the use of dolutegravir may potentially increase the risk of development of dolutegravir resistance. To date, studies on integrase (IN) genotypes of recently circulating viral strains before the use of INSTIs as well as pre-existing polymorphisms that may cause reduced sensitivity to INSTIs are lacking in Tanzania.

To address this, we recruited a total of 226 HIV-infected adults between June and October, 2017, at the HIV Care and Treatment Clinic in Muhimbili National Hospital, Dar es Salaam, Tanzania. Among them, 166 were viremic (plasma viral load $>400$ copies $/ \mathrm{mL}$ ) on first- or second-line regimen (not including INSTIs) for $>1$ year and the rest 60 were selfreported ART-naïve patients. The study protocol was approved by the Senate Research and Publications Committee of Muhimbili University of Health and Allied Sciences (Ref. No. 2016-12-07/AEC/Vol.XI/325) and the National Health Research Ethics Committee of the National Institute of Medical Research, Tanzania (NIMR/HQ/R.8a/Vol.IX/2373). Written informed consent was obtained from all study participants. Participant demographic and clinical information has been described previously. ${ }^{7}$

Whole blood was drawn from each study participant and the plasma fraction was separated by centrifugation within $2 \mathrm{~h}$ of sample collection. Viral RNA was then extracted from $140 \mu \mathrm{L}$ of the resultant plasma using the QIAamp Viral RNA Mini Kit, (QIAGEN) and used in one-step reverse transcrip- tion polymerase chain reaction (RT-PCR), followed by additional nested PCR, to amplify a part of the HIV-1 pol gene, as previously described. ${ }^{7}$ As a result, 866-base lengths of amplified DNA fragments encompassing IN were obtained from 47/60 ART-naïve and 111/166 viremic patients in ART treatment (not including INSTIs). These viral DNAs were directly sequenced by an automated sequencer (3500/3500XL genetic analyzer; Applied Biosystems). Sequence assembly was performed using SeqScape software, version 2.7, and each sequence was manually scrutinized to ensure sequence quality.

HIV-1 subtypes based on the IN region were first determined by the REGA HIV subtyping tool, v.3.0, as previously described. ${ }^{7}$ Unassigned sequences to a defined subtype were regarded as intersubtype recombinants. The results revealed that HIV subtypes A1 and C were the most predominant, accounting for $67 / 158(42 \%)$ and $60 / 158(38 \%)$, respectively. Other subtypes were D in $17 / 158(11 \%)$ patients and intersubtype recombinants in 14/158 (9\%) patients (Fig. 1A).

Phylogenetic analysis was also performed using HIV-1 sequences of IN regions. Sequences were aligned with reference to the HXB2 strain, and those that could not be aligned due to poor quality such as gaps and deletions were not included. Then, a phylogenetic tree was generated by using the PhyML interface tool (https://www.hiv.lanl.gov/content/ sequence/PHYML/interface.html). With the exception of intersubtype recombinants, majority of the sequences of specific groups of subtypes A1, C, and D, as determined by the REGA HIV subtyping tool, appropriately clustered together phylogenetically (Fig. 1B). The observation of cocirculating multiple subtypes and intersubtype recombinants in IN was consistent with previous reports analyzing protease (PR) and reverse transcriptase (RT) regions ${ }^{7}$ and represented a signature of the recent HIV-1 epidemic in Tanzania.

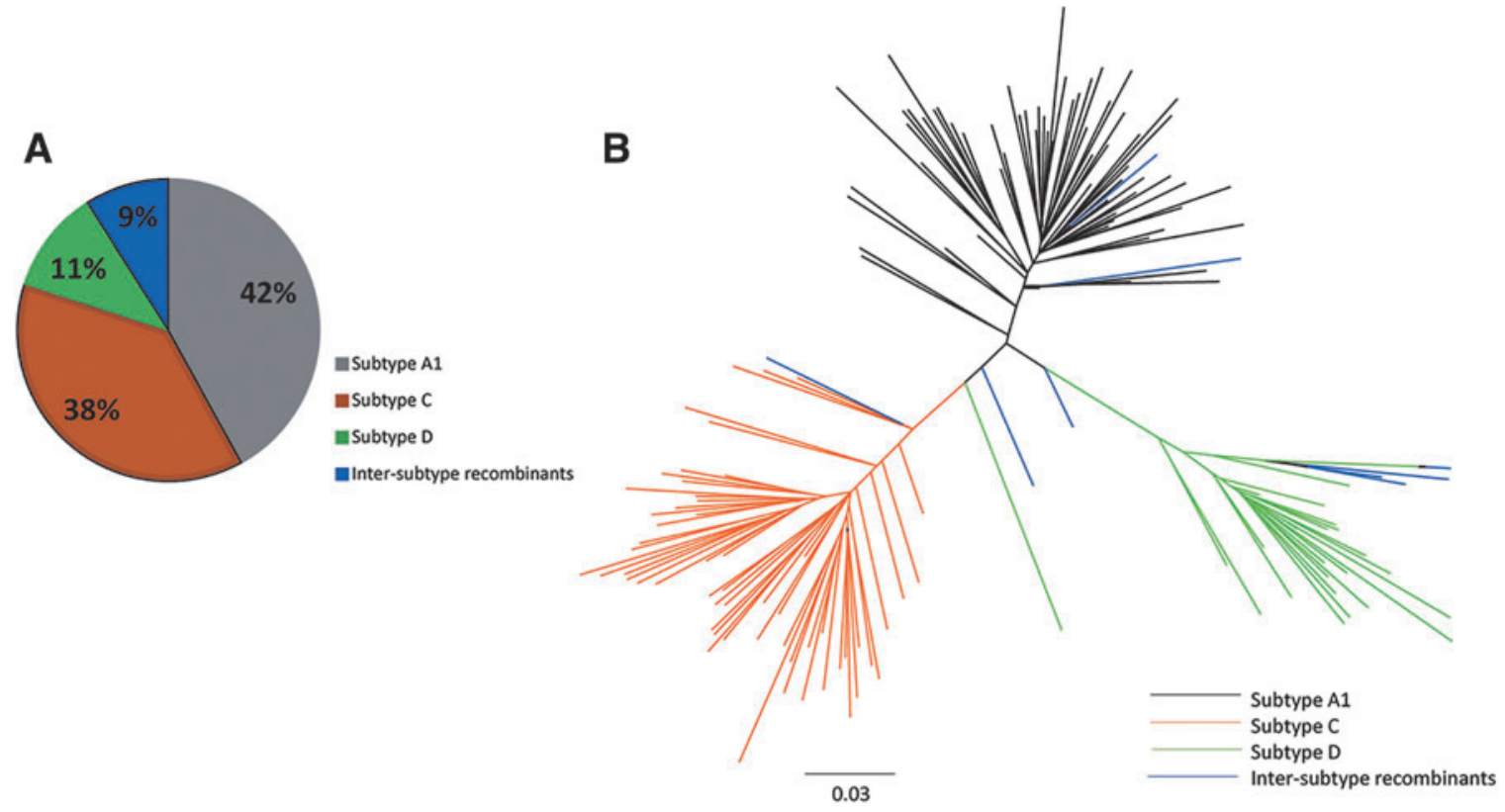

FIG. 1. The distribution of circulating HIV-1 subtypes among HIV-infected individuals in Tanzania. Pie chart indicates the frequency of HIV-1 subtypes circulating in Tanzania among $n=158$ study participants, as determined by the REGA HIV subtyping tool, version 3.0 (A). Phylogenetic tree shows the clustering patterns of HIV-1 circulating strains based on IN nucleotide sequences of HIV-1 (B). IN, integrase. 
We then analyzed the IN gene conservation pattern, as assessed by a cross-sectional measure of amino acid sequence variability in a population using the Shannon entropy score. ${ }^{8}$ The IN protein exhibited a relatively conserved pattern with an average entropy score of 0.16 , with $101 / 288$ (35\%) amino acid residues being highly conserved (Fig. 2A). In particular, two important functional motifs, His, His, Cys, and Cys at positions 12, 16, 40, and 42 as well as Asp, Asp, and Glu at positions 64,116 , and 154 , were $100 \%$ preserved with entropy scores of 0 . These observations were in agreement with previous reports 9 showing that these residues are important for IN function, structure, and viral fitness.

Amino acid conservation patterns were statistically significantly different across HIV subtypes $(p<.0001)$, with the highest average entropy score of 0.25 in subtype C (Fig. 2B). Interestingly, we observed that IN sequences from treated, but viremic, patients exhibited significantly greater conservation (entropy score of 0.14) compared with that of ARTnaïve patients (entropy score of 0.32$)(p<.0001)$ (Fig. 2C). However, this trend was not observed in viral PR-RT sequences from the same patients, whereby viral sequences from patients on treatment with NNRTI, NRTI, and PI exhibited a less conserved pattern compared with those from naive patients $(p=.008)$ (Fig. 2D). It is of interest to see whether the population-level amino acid variation or conservation in IN may change after introduction of INSTIs.

Next, the drug resistance genotype was assessed using the Stanford University HIV Drug Resistance Database, HIVdb Program, version 8.6.1 (https://hivdb.stanford.edu/hivdb). We found that none of the patients' sequences harbored any major INSTI resistance mutations. However, we detected amino acid variations at four positions (T97A, E157Q, G163E/K, and A128T) (Table 1) that had been reported previously as INSTI-resistance mutations. ${ }^{10}$ Mutation T97A was detected in 3/158 (1.8\%) patients in the present study. This mutation alone seems to have no effect on dolutegravir; however, it has been reported that T97A, in combination with other mutations, reduces virus susceptibility to dolutegravir. ${ }^{11} \mathrm{E} 157 \mathrm{Q}$, which was detected in $3 / 158$ (1.8\%) patients in our study, has been reported to have no impact on phenotypic susceptibility to dolutegravir when expressed alone. ${ }^{12}$ However, when E157Q is expressed in combination with R263K, it has been reported to increase dolutegravir resistance mediated by $\mathrm{R} 263 \mathrm{~K}$. $^{13}$

The importance of naturally occurring polymorphisms at codons 97 and 157 in the subsequent development of
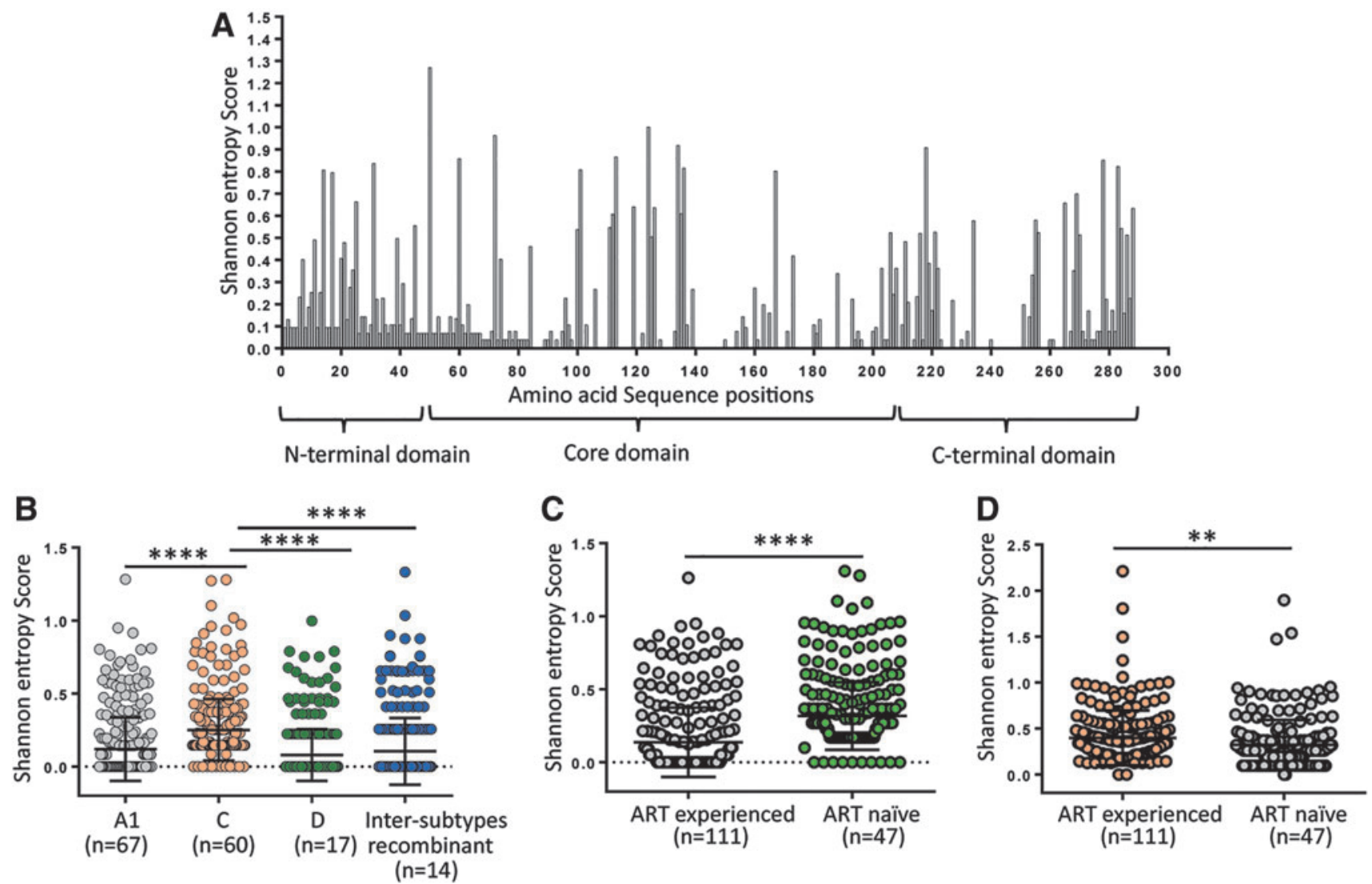

FIG. 2. Comparison of amino acid conservation patterns according to IN functional domains, HIV subtypes, and treatment status. The figure depicts entropy scores for each amino acid position $(n=288)$ in IN, the corresponding functional domains of the IN protein are indicated (A). The comparison of mean entropy scores for HIV subtypes A1, C, and D and intersubtype recombinants (B). The comparison of mean entropy scores of viral amino acid sequences from treatment (ART)experienced and treatment-naïve patients for IN (C) and PR-RT (D) regions. The horizontal lines indicate mean \pm standard deviation in the dot plots. $t$-Test with Bonferroni correction (B). $p$-Value of $<.05$ was considered statistically significant, and $* * * *$ and $* *$ indicate $p<.0001$ and $p<.001$, respectively. ART, antiretroviral therapy; PR, protease; RT, reverse transcriptase. 
Table 1. The Distribution of Integrase Inhibitor Accessory Drug Resistance Mutations Detected IN $N=8$ PATIENTS

\begin{tabular}{|c|c|c|c|c|c|c|c|}
\hline Patient ID & Treatment status & $\begin{array}{l}\text { HIV-1 } \\
\text { subtype }\end{array}$ & $\begin{array}{l}\text { INI major } \\
\text { resistance } \\
\text { mutations }\end{array}$ & $\begin{array}{l}\text { INI accessory } \\
\text { resistance } \\
\text { mutations }\end{array}$ & $\begin{array}{c}\text { NNRTI } \\
\text { mutations }\end{array}$ & $\begin{array}{l}\text { NRTI } \\
\text { mutations }\end{array}$ & $\begin{array}{l}\text { Protease } \\
\text { inhibitor } \\
\text { mutations }\end{array}$ \\
\hline V01 & Treat & A & - & T97TA & - & - & - \\
\hline V08 & Treatment naive & D & - & T97A & E138A & - & - \\
\hline V10 & Treatment naïve & RF C & - & E157Q & - & - & - \\
\hline V60 & Treatment naïve & A1 & - & T97A & - & - & - \\
\hline $\mathrm{X} 73$ & Virological failure & A1 & - & G163EK & $\begin{array}{l}\text { V106A, Y181C, } \\
\text { H221Y, F227L }\end{array}$ & D67G, M184V & - \\
\hline X81 & Virological failure & $\mathrm{D}$ & - & E157Q & $\begin{array}{l}\text { Y181C, H221Y, } \\
\text { F227L }\end{array}$ & $\begin{array}{l}\text { M41L, E44D, } \\
\text { M184V, L210W, } \\
\text { T215F }\end{array}$ & M46I, L76V \\
\hline X111 & Virological failure & $\mathrm{C}$ & - & E157Q & $\begin{array}{l}\text { K103N, V108I, } \\
\text { K238T }\end{array}$ & $\begin{array}{l}\text { M41L, M184V, } \\
\text { L210W, T215Y }\end{array}$ & - \\
\hline $\mathrm{X} 165$ & Virological failure & $\mathrm{C}$ & - & A128AT & - & - & - \\
\hline
\end{tabular}

INI, integrase inhibitor; NNRTI, non-nucleoside reverse transcriptase inhibitor; NRTI, nucleoside/nucleotide reverse transcriptase inhibitor; RF CD, recombinant form of subtypes C and D.

resistance to dolutegravir in Tanzanian settings should therefore be investigated with strategic longitudinal studies. Whereas G163E/K and A128T mutations were (rarely) observed only in $1 / 158(0.6 \%)$ patients, these two mutations have not so far been associated with virological failure in dolutegravir treatment-experienced patients. However, G163E/K appears to occur in combination with other integrase inhibitor (INI) resistance mutations, particularly $\mathrm{N} 155 \mathrm{H}$. A128T is referred as a miscellaneous INSTI-associated mutation that alone does not reduce INSTI susceptibility.

We finally analyzed the nucleotide sequences in the INencoding region for implications of the genetic barrier toward acquiring INSTI resistance mutations by assessing codon utilization at amino acid positions that are known to confer INSTI resistance. The genetic barrier was calculated with the sum of scores (the smallest number of transitions and transversions scored as 1.0 and 2.5, respectively) obtained for each amino acid position, as previously described. ${ }^{14} \mathrm{We}$ found that in almost all codons in our patient, IN sequences utilized a similar codon $(>90 \%)$ to that of global subtype-specific consensus sequences.

The observed minor variation in codon utilization did not result in a lower genetic barrier to drug resistance relative to global subtype-specific consensus sequences. This was also true at the codon positions $(118,155,230$, and 263) that have recently been associated with in vivo selection of dolutegravir resistance mutations (Table 2). Overall, our findings indicate that the genetic barrier to IN resistance in circulating IN sequences in Tanzania is mostly comparable with that of global HIV consensus, providing limited evidence of predisposition of dolutegravir resistance-prone polymorphisms in this country.

In conclusion, the present study has revealed the relatively conserved nature, yet unique patterns within a subtype and treatment status of patients, of the IN gene sequences in HIV-1 variants circulating in Tanzania despite multiple cocirculating subtypes. More importantly, the present study

Table 2. Codon Distribution and Calculated Genetic Barrier at Four Integrase Positions Associated with Selection of Dolutegravir Resistance Mutations In Vivo

\begin{tabular}{|c|c|c|c|c|c|c|c|c|}
\hline \multirow{2}{*}{$\begin{array}{l}\text { Codon } \\
\text { position }\end{array}$} & \multirow{2}{*}{$\begin{array}{l}\text { Resistance-associated } \\
\text { substitution }\end{array}$} & \multirow{2}{*}{$\begin{array}{l}\text { Codon } \\
\text { utilized }\end{array}$} & \multicolumn{4}{|c|}{ \% Codon utilization per HIV subtype } & \multirow{2}{*}{$\begin{array}{c}\text { Resistance-associated } \\
\text { codon }\end{array}$} & \multirow{2}{*}{$\begin{array}{l}\text { Lower } \\
\text { score }\end{array}$} \\
\hline & & & $A$ & $C$ & $D$ & $R F$ & & \\
\hline \multirow[t]{6}{*}{118} & \multirow[t]{6}{*}{ G118R } & GGC & 82.7 & 95.6 & 100 & 88.4 & AGC & 1 \\
\hline & & GGA & 1.7 & 0 & 0 & 0 & AGA & 1 \\
\hline & & GGT & 10.3 & 2.2 & 0 & 4.6 & AGT & 1 \\
\hline & & GGR & 1.7 & 0 & 0 & 0 & & \\
\hline & & GGW & 3.4 & 0 & 0 & 0 & & \\
\hline & & GGY & 1.7 & 2.2 & 0 & 7 & & \\
\hline \multirow[t]{3}{*}{155} & \multirow[t]{3}{*}{$\mathrm{N} 155 \mathrm{H}$} & AAT & 95.6 & 96.6 & 100 & 88.1 & CAT & 2.5 \\
\hline & & $\mathrm{AAC}$ & 4.3 & 1.7 & 0 & 7.1 & CAC & 2.5 \\
\hline & & AAY & 0 & 1.7 & 0 & 4.8 & & \\
\hline \multirow[t]{2}{*}{230} & \multirow[t]{2}{*}{ S230R } & $\mathrm{AGC}$ & 100 & 97.8 & 100 & 100 & CGC & 2.5 \\
\hline & & AAC & 0 & 2.2 & 0 & 0 & CGC & 3.5 \\
\hline \multirow[t]{3}{*}{263} & \multirow[t]{3}{*}{$\mathrm{R} 263 \mathrm{~K}$} & $\mathrm{AGA}$ & 91.3 & 13.8 & 83.3 & 66.7 & AAA & 1 \\
\hline & & AGG & 6.5 & 79.2 & 16.7 & 28.6 & AAG & 1 \\
\hline & & AGR & 2.1 & 7 & 0 & 4.8 & & \\
\hline
\end{tabular}


clearly demonstrated the lack of major IN resistance mutations and a low frequency of naturally occurring polymorphisms that may confer resistance to INSTIs, similar to other African countries. ${ }^{15}$ Therefore, dolutegravir should be effective in these countries, including Tanzania. However, strategic longitudinal studies of T97T/A and E157Q polymorphisms in the subsequent development of resistance to dolutegravir in Tanzanian settings should be investigated, and continuous surveillance of INSTI resistance genotypes after initiation of the INI is warranted.

\section{Sequence Data}

GenBank accession numbers for 158 nucleotide sequences of IN are MT060493-MT060651.

\section{Author Disclosure Statement}

No competing financial interests exist.

\section{Funding Information}

This study was supported, in part, by a grant from the Japan Agency for Medical Research and Development, AMED (Research Program on HIV/AIDS), JSPS KAKENHI Grantin-Aid for Scientific Research, JSPS Bilateral Open Partnership Joint Research Projects, and JSPS Core-to-Core Program. M.M. received funding from the Terumo Foundation for Life Sciences and Arts. G.B. is supported by the scholarship for The International Priority Graduate Programs; Advanced Graduate Courses for International Students (Doctoral Course), MEXT, Japan. The funders had no role in study design, data collection and interpretation, or the decision to submit the work for publication.

\section{References}

1. Rutstein SE, Chen JS, Nelson JAE, Phiri S, Miller WC, Hosseinipour MC: High rates of transmitted NNRTI resistance among persons with acute HIV infection in Malawi: Implications for first-line dolutegravir scale-up. AIDS Research and Therapyvolume 2019;16:1623.

2. Wittkop L, Günthard HF, de Wolf F, et al: : Effect of transmitted drug resistance on virological and immunological response to initial combination antiretroviral therapy for HIV (EuroCoord-CHAIN joint project): A European multicohort study. Lancet Infect Dis 2011;11: 363-371.

3. Phillips AN, Venter F, Havlir D, et al.: Risks and benefits of dolutegravir-based antiretroviral drug regimens in subSaharan Africa: A modelling study. Lancet HIV 2019;6: e116-e127.

4. Max B, Vibhakar S: Dolutegravir: A new HIV integrase inhibitor for the treatment of HIV infection. Fut Virol 2014; 9:967-978.

5. Seatla K, Avalos A, Moyo S, et al:: Four-class drugresistant HIV-1 subtype $\mathrm{C}$ in a treatment experienced in- dividual on dolutegravir-based antiretroviral therapy in Botswana. AIDS 2018;32:1899-1902.

6. Brenner BG, Lowe M, Moisi D, et al.: Subtype diversity associated with the development of HIV-1 resistance to integrase inhibitors. J Med Virol 2011;83:751-759.

7. Barabona G, Mahiti M, Masoud S, et al.: Pre-treatment and acquired HIV drug resistance in Dar es Salaam, Tanzania in the era of tenofovir and routine viral load monitoring. J Antimicrob Chemother 2019;74:3016-3020.

8. Yusim K, Kesmir C, Gaschen B, et al.: Clustering patterns of cytotoxic T-lymphocyte epitopes in human immunodeficiency virus type 1 (HIV-1) proteins reveal imprints of immune evasion on HIV-1 global variation. J Virol 2002; 76:8757-8768.

9. Bessong PO: Polymorphisms in HIV-1 subtype C proteases and the potential impact on protease inhibitors. Trop Med Int Health 2008;13:144-151.

10. Mboumba Bouassa RS, Mossoro-Kpinde CD, Gody JC, et al:: High predictive efficacy of integrase strand transfer inhibitors in perinatally HIV-1-infected African children in therapeutic failure of first- and second-line antiretroviral drug regimens recommended by the WHO. J Antimicrob Chemother 2019;74:2030-2038.

11. Mascolini M: High Level Resistance to Dolutegravir (DTG) after emergence of T97A mutation. Available at www.natap.org/2018/CROI/croi_140.htm (2018), accessed August 27, 2019.

12. Charpentier C, Malet I, Andre-Garnier E, et al.: Phenotypic analysis of HIV-1 E157Q integrase polymorphism and impact on virological outcome in patients initiating an integrase inhibitor-based regimen. J Antimicrob Chemother 2018;73:1039-1044.

13. Rhee SY, Grant PM, Tzou PL, et al.: A systematic review of the genetic mechanisms of dolutegravir resistance. J Antimicrob Chemother 2019;74:3135-3149.

14. Van de Vijver DA, Wensing A, Angarano G, et al:: The calculated genetic barrier for antiretroviral drug resistance substitution is largely similar for different HIV-1 subtypes. J Acquir Immune Defic Syndr 2006;41:352-360.

15. Doyle T, Dunn DT, Ceccherini-Silberstein F, et al.: Integrase inhibitor (INI) genotypic resistance in treatmentnaive and raltegravir-experienced patients infected with diverse HIV-1 clades. J Antimicrob Chemother 2015;70: 3080-3086.

Address correspondence to: Takamasa Ueno Division of Infection and Immunity Joint Sciences Research Center for Human Retrovirus Infection Kumamoto University 2-2-1 Chuo-ku

Honjo, Kumamoto 860-0811 Japan

E-mail: uenotaka@kumamoto-u.ac.jp 